\title{
Heterogeneous Impatience in a Continuous-Time Model
}

\author{
Chiaki Hara* \\ Institute of Economic Research, Kyoto University
}

October 25, 2007

\begin{abstract}
In a continuous-time economy with complete markets, we show how the heterogeneity in the individual consumers' risk attitudes and impatience would affect the representative consumer's counterparts. Specifically, our formulas tell us how his risk tolerance and impatience will change over time, and how his impatience will be affected by the changes in aggregate consumption levels. Under the assumption of equal and constant relative risk aversion across individual consumers, we characterize his discount factor by means of a completely monotone function of time. These results are used to analyze short-rate processes.
\end{abstract}

JEL Classification Codes: D51, D53, D61, D81, D91, G12, G13.

Keywords: Representative consumer, expected utility, time additivity, multiplicative separability, impatience, risk tolerance, state-price deflator, short-rate process, complete monotonicity.

\section{Introduction}

In this paper, we consider a dynamic economic model of continuous-time consisting of multiple consumers, rather than a single representative consumer. Our purpose is to assess the impacts of heterogeneity of consumers' impatience (often measured as discount rates) and risk attitudes (often measured as absolute risk aversion or its reciprocal, absolute risk tolerance) on equilibrium asset pricing. It is well known that if the asset markets are complete, we can define a representative consumer as the value function of the problem of maximizing a weighted sum of individual consumers' utilities and that we can use his marginal utilities, evaluated at the aggregate consumption process, as the state-price deflator to price all assets at any point in time. Then the task of investigating the impacts of heterogeneous impatience and risk attitudes on asset prices boils down to the task of investigating the impacts of heterogeneous impatience

\footnotetext{
*I am grateful to helpful comments from members of the Grant in Aid for Specially Promoted Research from Japan Society for the Promotion of Sciences for "Economic Analysis on Intergenerational Problems". The grant is also gratefully acknowledged. I have also received a generous research grant from Ishii Memorial Foundation for the Promotion of Financial Studies on "Microeconomic Foundations of the Term Structure of Interest Rates". I received helpful comments from the Microeconomic Theory Workshop at the Institute of Economic Research of Kyoto University, and also from Masaaki Kijima and Katsumasa Nishide. My email address is hara@kier.kyoto-u.ac.jp.
} 
and risk attitudes on the representative consumer's utility function. This is what we shall do in this paper.

We aim at establishing general properties of the representative consumer's utility function arising from heterogeneity. By being general, what we mean here is that the results we are after ought to be independent of the number of (types of) consumers in the economy, the functional forms of their utility (or felicity) functions, and the stochastic characteristics of the consumption processes.

We also aim at establishing some formulas describing how the heterogeneity affects the representative consumer's utility function. To see why obtaining such a formula, as opposed to a merely qualitative prediction, is useful, let us compare one of our results (Theorem 3 and its corollary, Corollary 3) with an existing one (Proposition 5 of Gollier and Zeckhauser (2005)). Proposition 5 of Gollier and Zeckhauser (2005) showed that if all consumers have constant but unequal impatience (so that the discount factor is a negative exponential function of time) and exhibit decreasing absolute risk aversion, then the representative consumer's impatience will decrease over time. On the other hand, Theorem 3 and Corollary 3 give formulas that relate the individual consumers' impatience and cautiousness (the derivative of absolute risk tolerance) to the derivative of the representative consumer's impatience with respect to time. One can easily see from our formulas that as long as there are many consumers, the representative consumer's impatience would decrease over time even if the assumption is decreasing absolute risk aversion is violated just by one consumer (or by a small number of consumers). But the qualitative prediction by Gollier and Zeckhauser, as it stands, does not allow us to judge whether the prediction would still be true when the deviations are small. In short, while formulas allow us to see that some conclusions are robust to small deviations from the assumptions, qualitative predictions do not. This is why we aim at obtaining formulas rather than just qualitative predictions.

Throughout the paper, we assume that all consumers' utility functions are time-additive. Although this is a fairly common assumption in finance and macroeconomics, it does exclude some utility functions, such as recursive utilities and utilities of habit formation. By excluding them, we do not mean that they are unimportant or uninteresting. Rather, our intention is to make full use of the existing analytical techniques on the impacts of heterogeneous risk attitudes, as presented in Hara, Huang, and Kuzmics (2007), under the assumption of expected utilities and equal impatience, to the analysis of heterogeneous impatience.

This paper is most closely related to Gollier and Zeckhauser (2005). They investigated how the representative consumer's impatience is affected by the heterogeneity of the individual consumers' impatience. They also showed that if there are infinitely many consumers and their impatience are exponentially distributed, then the representative consumer may exhibit hyperbolic discounting. But their model is a deterministic one and, as discussed above, their results tends to be qualitative. In a discrete-time model under uncertainty, Malamud and Trubowitz (2006) showed that the way in which the representative consumer's risk aversion changes over time is determined by the covariance of the individual consumers' impatience and 
cautiousness. In Sections 3 and 4, we use a similar approach to obtain more general results with simpler proofs. The methodology of this paper closely follows that of Hara, Huang, and Kuzmics (2007). Hara (2006) applied the techniques of Hara, Huang, and Kuzmics (2007) to a continuous-time setup under the assumption that all consumers have the discount rate. This paper can thus be considered as an extension of that paper to the case of unequal impatience.

An important message of the results in this paper is that if the representative consumer is truly representative, in the sense that his utility function is derived from an explicitly modeled group of heterogeneous consumers via the social welfare maximization problem, then his utility function is quite likely to exhibit decreasing discount rates and unlikely to be multiplicatively separable. This fact cast serious doubts on the plausibility of the prevalent use of constant discount rates and multiplicatively separable utility functions for the representative consumer in the representative-consumer models of dynamic macroeconomics.

This paper is organized as follows: In Section 2, we lay out the setup of this paper and explain basic concepts for our analysis. In Section 3, we identify the implications of heterogeneous impatience and risk attitudes on the representative consumer's risk attitudes. In Section 4, we identify the implications on his impatience. In Section 5, we summarize our results and suggest two directions of future research.

\section{Setup}

The setup of this paper is as follows. The economy is subject to uncertainty, which is represented by a probability measure space $(\Omega, \mathscr{F}, P)$. The time span is $\boldsymbol{R}_{+}=[0, \infty)$, which is of continuous time and infinite length, although it could be $[0, T]$ with $0<T<\infty$, which is of finite length. The gradual information revelation is represented by a filtration $\left(\mathscr{F}_{t}\right)_{t \in \boldsymbol{R}_{+}}$. There is only one type of good on each time and state.

The economy consists of $I$ consumers. Each consumer $i$ has a possibly time-dependent felicity function $u_{i}: \boldsymbol{R}_{++} \times \boldsymbol{R}_{+} \rightarrow \boldsymbol{R}$, which is continuously differentiable in both variables and at least twice continuously differentiable in the first variable (consumption level), ${ }^{1}$ and satisfies $\partial u_{i}\left(x^{i}, t\right) / \partial x^{i}>0>\partial^{2} u_{i}\left(x^{i}, t\right) / \partial\left(x^{i}\right)^{2}$ for every $\left(x^{i}, t\right) \in \boldsymbol{R}_{++} \times \boldsymbol{R}_{+}$and the Inada condition, that is, for every $t \in \boldsymbol{R}_{+}, \partial u_{i}\left(x^{i}, t\right) / \partial x^{i} \rightarrow 0$ as $x^{i} \rightarrow \infty$, and $\partial u_{i}\left(x^{i}, t\right) / \partial x^{i} \rightarrow \infty$ as $x^{i} \rightarrow 0$. His utility function $U_{i}$ over stochastic consumption processes are then defined by requiring time additivity and state-independent expected utility:

$$
U_{i}\left(c^{i}\right)=E\left(\int_{0}^{\infty} u_{i}\left(c_{t}^{i}, t\right) d t\right)
$$

where $c^{i}=\left(c_{t}^{i}\right)_{t \in \boldsymbol{R}_{+}}$is an adapted process taking values in $\boldsymbol{R}_{++} \cdot{ }^{2}$

The key parameters of the felicity function $u_{i}$ (and thus of the utility function $U_{i}$ ) are risk

\footnotetext{
${ }^{1}$ The degree of continuous differentiability needed in each of the subsequent results will be made clear in its proof.

${ }^{2}$ To be exact, we need to impose some additional restrictions on $c^{i}$ to make the integral well defined (finite). As such restrictions are irrelevant to the subsequent analysis, we shall not explicitly state or impose them.
} 
tolerance and impatience. The risk tolerance $s_{i}: \boldsymbol{R}_{++} \times \boldsymbol{R}_{+} \rightarrow \boldsymbol{R}_{++}$is defined by

$$
s_{i}\left(x^{i}, t\right)=-\frac{\partial u_{i}\left(x^{i}, t\right) / \partial x^{i}}{\partial^{2} u_{i}\left(x^{i}, t\right) / \partial\left(x^{i}\right)^{2}} .
$$

This is nothing but the reciprocal of the Arrow-Pratt measure of absolute risk aversion. In this dynamic setup, this also measures tolerance to intertemporal consumption fluctuations. The partial derivative with respect to $x_{i}, \partial s_{i}\left(x_{i}, t\right) / \partial x_{i}$, is called the cautiousness. The impatience, or discount rate, $r_{i}: \boldsymbol{R}_{++} \times \boldsymbol{R}_{+} \rightarrow \boldsymbol{R}$ is defined by

$$
r_{i}\left(x^{i}, t\right)=-\frac{\partial^{2} u_{i}\left(x^{i}, t\right) / \partial x^{i} \partial t}{\partial u_{i}\left(x^{i}, t\right) / \partial x^{i}} .
$$

An important class of utility functions is one of multiplicatively separable utility functions. A utility function $u_{i}$ is multiplicatively separable if there are two functions $v_{i}: \boldsymbol{R}_{++} \rightarrow \boldsymbol{R}$ and $d_{i}: \boldsymbol{R}_{+} \rightarrow \boldsymbol{R}_{++}$such that $u_{i}\left(x^{i}, t\right)=d_{i}(t) v_{i}\left(x^{i}\right)$ for every $\left(x^{i}, t\right) \in \boldsymbol{R}_{++} \times \boldsymbol{R}_{+}$. Then $s_{i}\left(x^{i}, t\right)=-v_{i}^{\prime}\left(x^{i}\right) / v_{i}^{\prime \prime}\left(x^{i}\right)$ and $r_{i}\left(x^{i}, t\right)=-d_{i}^{\prime}(t) / d_{i}(t)$. We thus write $s_{i}\left(x_{i}\right)$ for $s_{i}\left(x^{i}, t\right)$ and $r_{i}(t)$ for $r_{i}\left(x^{i}, t\right)$ in this case. Then

$$
\frac{d_{i}(t)}{d_{i}(0)}=\exp \left(-\int_{0}^{t} r_{i}(\tau) d \tau\right)
$$

for every $t$. If, in addition, there exists a $\rho_{i}>0$ such that $d_{i}(t)=\exp \left(-\rho_{i} t\right)$, then $r_{i}(t)=\rho_{i}$ for every $t \in \boldsymbol{R}_{+}$. This is the case of exponential discounting.

To find a Pareto efficient allocation of a given aggregate consumption process $c=\left(c_{t}\right)_{t \in \boldsymbol{R}_{+}}$ and its supporting (decentralizing) state-price deflator, it is sufficient to choose positive numbers $\lambda_{1}, \ldots, \lambda_{I}$ and consider the following maximization problem:

$$
\begin{array}{cl}
\max _{\left(c^{1}, \ldots, c^{I}\right)} & \sum_{i} \lambda_{i} U_{i}\left(c^{i}\right) \\
\text { subject to } & \sum_{i} c^{i}=c .
\end{array}
$$

Since the utility functions $U_{i}$ are additive with respect to both time and states and the probabilistic belief $P$ is common across consumers, it can be rewritten as

$$
\sum_{i} \lambda_{i} U_{i}\left(c^{i}\right)=E\left(\int_{0}^{\infty} \sum_{i} \lambda_{i} u_{i}\left(c_{t}^{i}, t\right) d t\right) .
$$

Hence, to solve the maximization problem (1), it suffices to solve

$$
\begin{array}{ll}
\underset{\begin{array}{l}
\left.\max _{1}, \ldots, x_{I}\right) \in \boldsymbol{R}_{++}^{I} \\
\text { subject to }
\end{array}}{ } & \sum_{i} \lambda_{i} u_{i}\left(x^{i}, t\right) \\
& \sum_{i} x^{i}=x .
\end{array}
$$

for each pair of a realized aggregate consumption level $x \in \boldsymbol{R}_{++}$and time $t \in \boldsymbol{R}_{+}$. It can be 
easily proved that under the stated conditions, there is a unique solution, which we denote by $\left(f_{1}(x, t), \ldots, f_{I}(x, t)\right)$. It can also be shown that for each $f_{i}$ is continuously differentiable in both variables. We can define the value function of this problem $u: \boldsymbol{R}_{++} \times \boldsymbol{R}_{+} \rightarrow \boldsymbol{R}$ by

$$
u(x, t)=\sum_{i} \lambda_{i} u_{i}\left(f_{i}(x, t), t\right)
$$

This is the felicity function of the representative consumer. It need not be multiplicatively separable between time $t$ and the consumption level $x$ when all individual consumers discounts future utilities exponentially but at differing rates. The representative consumer's utility function is

$$
U(c)=E\left(\int_{0}^{\infty} u\left(c_{t}, t\right) d t\right)
$$

Just as for an individual consumer's utility function, we define risk tolerance and impatience as follows:

$$
s(x, t)=-\frac{\partial u(x, t) / \partial x}{\partial^{2} u_{i}(x, t) / \partial x^{2}} \text { and } r(x, t)=-\frac{\partial^{2} u_{i}(x, t) / \partial x \partial t}{\partial u(x, t) / \partial x} .
$$

The cautiousness is defined as the partial derivative $\partial s(x, t) / \partial x$ with respect to the aggregate consumption level $x$.

The representative consumer is, of course, not an "actual" consumer, who would trade on financial markets. Rather, he is a theoretical construct, whom we can use to identify asset prices. Specifically, if $u$ is the representative consumer's felicity function and $c=\left(c_{t}\right)_{t \in \boldsymbol{R}_{+}}$is the aggregate consumption process, then his marginal utility process evaluated at the aggregate consumption, $\left(\partial u\left(c_{t}, t\right) / \partial x\right)_{t \in \boldsymbol{R}_{+}}$, is the state price process. This means that the price at time $t$ of an asset with dividend (rate) process $\delta=\left(\delta_{t}\right)_{t \in \boldsymbol{R}_{+}}$is equal to the discounted sum of its future dividends:

$$
E\left(\int_{t}^{\infty} \frac{\partial u\left(c_{\tau}, \tau\right) / \partial x}{\partial u\left(c_{t}, t\right) / \partial x} \delta_{\tau} d t\right)
$$

Moreover, if the aggregate consumption process is defined by an Ito integral, then the short-rate process, which keeps track of interest rates for risk-free lending and borrowing for infinitesimally short intervals of time, can be represented in terms of the representative consumer's risk tolerance $s$ and impatience $r$, and the drift and diffusion terms of the aggregate consumption process.

Although we analyze the Pareto efficient allocations, if the asset markets are complete, then our analysis is applicable to the equilibrium allocations and asset prices. This is because the first welfare theorem holds in complete markets, so that the equilibrium allocations are Pareto efficient and the equilibrium asset prices are given by the marginal utility process. Since the $u_{i}(\cdot, t)$ are concave, the second welfare theorem also holds, so that every Pareto efficient allocation is an equilibrium allocation for some distribution of initial endowments. Hence an analysis of Pareto efficient allocations is also an analysis of equilibrium allocations.

The solution to the maximization problem (1) is a Pareto efficient allocation. When it is an equilibrium allocation, the individual consumers' wealth shares, evaluated by the equilibrium 
prices, are positively related to the utility weights $\lambda_{i}$ in (1). All the properties we shall explore in the subsequent analysis are valid regardless of the choice of utility weights. Hence, these properties are also valid for the equilibrium allocations regardless of wealth distributions.

To develop our analysis, we now list up some of the results that have been in the existing literature and hold for every $(x, t) \in \boldsymbol{R}_{++} \times \boldsymbol{R}_{+}$. By Theorems 4 and 5 of Wilson (1968),

$$
\begin{aligned}
s(x, t) & =\sum_{i} s_{i}\left(f_{i}(x, t), t\right), \\
\frac{\partial f_{i}}{\partial x}(x, t) & =\frac{s_{i}\left(f_{i}(x, t), t\right)}{s(x, t)} .
\end{aligned}
$$

By differentiating both sides of (3) with respect to $x$ and applying (4), we obtain

$$
\frac{\partial s}{\partial x}(x, t)=\sum_{i} \frac{\partial f_{i}}{\partial x}(x, t) \frac{\partial s_{i}}{\partial x^{i}}\left(f_{i}(x, t), t\right)=\sum_{i} \frac{s_{i}\left(f_{i}(x, t), t\right)}{s(x, t)} \frac{\partial s_{i}}{\partial x^{i}}\left(f_{i}(x, t), t\right)
$$

This shows that the representative consumer's cautiousness is the weighted average of the individual consumers' counterparts, where the weights are proportional to their absolute risk tolerance.

By equality (10) and Proposition 3 of Gollier and Zeckhauser (2005),

$$
\begin{aligned}
r(x, t) & =\sum_{i} \frac{s_{i}\left(f_{i}(x, t), t\right)}{s(x, t)} r_{i}\left(f_{i}(x, t), t\right) \\
\frac{\partial f_{i}}{\partial t}(x, t) & =s_{i}\left(f_{i}(x, t), t\right)\left(r(x, t)-r_{i}\left(f_{i}(x, t), t\right)\right) .
\end{aligned}
$$

(6) means that the representative consumer's impatience is the weighted average of the individual consumers' counterparts where the weights are proportional to their absolute risk tolerance. (7) means that if an individual consumer is more patient than the representative consumer, the former's consumption level would grow over time were the aggregate consumption level to be constant, and the growth rate is proportional to his absolute risk tolerance.

By applying Theorem 4 of Hara, Huang, and Kuzmics (2006) to the $u_{i}(\cdot, t)$ for a fixed $t$ and using (4), we can obtain

$$
\begin{aligned}
\frac{\partial^{2} s}{\partial x^{2}}(x, t)= & \sum_{i}\left(\frac{s_{i}\left(f_{i}(x, t), t\right)}{s(x, t)}\right)^{2} \frac{\partial^{2} s_{i}}{\partial\left(x^{i}\right)^{2}}\left(f_{i}(x, t), t\right) \\
& +\frac{1}{s(x, t)} \sum_{i} \frac{s_{i}\left(f_{i}(x, t)\right)}{s(x, t)}\left(\frac{\partial s_{i}}{\partial x^{i}}\left(f_{i}(x, t), t\right)-\frac{\partial s}{\partial x}(x, t)\right)^{2} .
\end{aligned}
$$

To understand this formula, note first that by (4), the first term on the right-hand side can be written as

$$
\sum_{i} \frac{s_{i}\left(f_{i}(x, t), t\right)}{s(x, t)}\left(\frac{\partial^{2} s_{i}}{\partial\left(x^{i}\right)^{2}}\left(f_{i}(x, t), t\right) \frac{\partial f_{i}}{\partial x}(x, t)\right)
$$

Here, the term $\left(\partial^{2} s_{i}\left(f_{i}(x, t), t\right) / \partial\left(x^{i}\right)^{2}\right)\left(\partial f_{i}(x, t) / \partial x\right)$ is the change in the cautiousness of 
consumer $i$ arising from the increase in his consumption level, which is, in turn, caused by an increase in the aggregate consumption level. Thus the first term represents the direct effect on the representative consumer's cautiousness by an increase in aggregate consumption. By (3), the second term on the right-hand side is the weighted variance of the individual consumers' cautiousness, divided by the representative consumer's absolute risk aversion. This formula therefore shows that the heterogeneity in the individual consumers' cautiousness increases the representative consumer's cautiousness, thereby making his risk tolerance, as a function of aggregate consumption levels, more convex.

\section{Representative Consumer's Risk Tolerance}

We are interested in how the representative consumer's risk tolerance and impatience vary over time when the individual consumers' counterparts are heterogeneous.

The first result of this paper is concerned with how the representative consumer's risk tolerance varies over time. In the special case of exponential discounting, an essentially identical formula was already given in the proof of Theorem 3.3 of Malamud and Trubowitz (2006).

Theorem 1 For every $(x, t) \in \boldsymbol{R}_{++} \times \boldsymbol{R}_{+}$,

$$
\begin{aligned}
\frac{\partial s}{\partial t}(x, t)= & \sum_{i} \frac{\partial s_{i}}{\partial t}\left(f_{i}(x, t), t\right) \\
& -s(x, t) \sum_{i} \frac{s_{i}\left(f_{i}(x, t)\right)}{s(x, t)}\left(\frac{\partial s_{i}}{\partial x^{i}}\left(f_{i}(x, t), t\right)-\frac{\partial s}{\partial x}(x, t)\right)\left(r_{i}\left(f_{i}(x, t)\right)-r(x, t)\right) .
\end{aligned}
$$

This theorem tells us that the rate, per unit of time, of changes in the representative consumer's risk tolerance can be decomposed into two terms. The first term on the right-hand side is easy to grasp. As shown by (3), the representative consumer's risk tolerance is the sum of the individual consumers' counterparts. Thus the first term represents the direct effect on risk tolerance by time. It is equal to zero when all individual consumers' felicity functions $u_{i}$ are multiplicatively separable.

By (5) and (6), the second term of (8) is equal to the weighted covariance, multiplied by the representative consumer's risk tolerance, between the individual consumers' cautiousness and impatience, where the weights are proportional to the individual consumers' risk tolerance. Since the second term would be zero if all consumers' cautiousness or impatience are equal to one another, it captures the tendency of changes in the representative consumer's impatience that arise from the heterogeneity in the individual consumers' cautiousness and impatience.

Combining the first and second terms of the right-hand side of (8), Theorem 1 states that the rate of changes, per unit of time, in the representative consumer's risk tolerance is the sum of the individual counterparts subtracted by the weighted covariance, multiplied by his own risk tolerance, between the individual consumers' cautiousness and impatience. 
Proof of Theorem 1 Differentiate both sides of (3) with respect to $t$, then we obtain

$$
\begin{aligned}
\frac{\partial s}{\partial t}(x, t) & =\sum_{i}\left(\frac{\partial s_{i}}{\partial x^{i}}\left(f_{i}(x, t), t\right) \frac{\partial f_{i}}{\partial t}(x, t)+\frac{\partial s_{i}}{\partial t}\left(f_{i}(x, t), t\right)\right) \\
& =\sum_{i} \frac{\partial s_{i}}{\partial t}\left(f_{i}(x, t), t\right)+\sum_{i} \frac{\partial s_{i}}{\partial x^{i}}\left(f_{i}(x, t), t\right) s_{i}\left(f_{i}(x, t), t\right)\left(r(x, t)-r_{i}\left(f_{i}(x, t), t\right)\right),
\end{aligned}
$$

where the last equality follows from (7). Since

$$
\sum_{i} s_{i}\left(f_{i}(x, t), t\right)\left(r(x, t)-r_{i}\left(f_{i}(x, t), t\right)\right)=0,
$$

the second term of (9) can be written as

$$
\sum_{i}\left(\frac{\partial s_{i}}{\partial x^{i}}\left(f_{i}(x, t), t\right)-\frac{\partial s}{\partial x}(x, t)\right) s_{i}\left(f_{i}(x, t), t\right)\left(r(x, t)-r_{i}\left(f_{i}(x, t), t\right)\right) .
$$

This is equal to the second term of the right-hand side of (8).

Theorem 1 has a couple of implications. The first one is a generalization of Theorem 3.3 of Malamud and Trubowitz (2006) to the case of multiplicatively separable utility functions.

Corollary 1 Suppose that $u_{i}$ is multiplicatively separable for every $i$. Then

$$
\frac{\partial s(x, t) / \partial t}{s(x, t)}=-\sum_{i} \frac{s_{i}\left(f_{i}(x)\right)}{s(x, t)}\left(s_{i}^{\prime}\left(f_{i}(x, t)\right)-\frac{\partial s}{\partial x}(x, t)\right)\left(r_{i}(t)-r(x, t)\right)
$$

for every $(x, t) \in \boldsymbol{R}_{++} \times \boldsymbol{R}_{+}$. Moreover,

1. If $\left(s_{1}^{\prime}\left(f_{1}(x, t)\right), \ldots, s_{I}^{\prime}\left(f_{I}(x, t)\right)\right)$ and $\left(r_{1}(t), \ldots, r_{I}(t)\right)$ are comonotone (that is, $\left(s_{i}^{\prime}\left(f_{i}(x, t)\right)-s_{j}^{\prime}\left(f_{j}(x, t)\right)\right)\left(r_{i}(t)-r_{j}(t)\right) \geq 0$ for every pair of two consumers $i$ and $\left.j\right)$, then $\partial s(x, t) / \partial t \leq 0$. This weak inequality holds as an equality if and only if $s_{1}^{\prime}\left(f_{1}(x, t)\right)=$ $\cdots=s_{I}^{\prime}\left(f_{I}(x, t)\right)$ or $r_{1}(t)=\cdots=r_{I}(t)$.

2. If $\left(s_{1}^{\prime}\left(f_{1}(x, t)\right), \ldots, s_{I}^{\prime}\left(f_{I}(x, t)\right)\right)$ and $\left(r_{1}(t), \ldots, r_{I}(t)\right)$ are anti-comonotone (that is, $\left(s_{i}^{\prime}\left(f_{i}(x, t)\right)-s_{j}^{\prime}\left(f_{j}(x, t)\right)\right)\left(r_{i}(t)-r_{j}(t)\right) \leq 0$ for every pair of two consumers $i$ and $\left.j\right)$, then $\partial s(x, t) / \partial t \geq 0$. This weak inequality holds as an equality if and only if $s_{1}^{\prime}\left(f_{1}(x, t)\right)=$ $\cdots=s_{I}^{\prime}\left(f_{I}(x, t)\right)$ or $r_{1}(t)=\cdots=r_{I}(t)$.

An important case of this corollary is Corollary 3.4 of Malamud and Trubowitz, which deals with constant relative risk aversion and exponential discounting. In this case, $s_{i}^{\prime}\left(x^{i}\right)$ is equal to the reciprocal of constant relative risk aversion and $r(t)$ is equal to the constant impatience, so that the validity of the assumption of (anti-)comonotonicity can be checked without reference to the choice of the consumption levels $x^{i}$ or time $t$. 


\section{Representative Consumer's Impatience}

In this section, we turn our attention to the representative consumer's impatience. The first result of this section is concerned with how the representative consumer's impatience is affected by aggregate consumption levels.

Theorem 2 For every $(x, t) \in \boldsymbol{R}_{++} \times \boldsymbol{R}_{+}$,

$$
\begin{aligned}
\frac{\partial r}{\partial x}(x, t)= & \sum_{i}\left(\frac{s_{i}\left(f_{i}(x, t), t\right)}{s(x, t)}\right)^{2} \frac{\partial r_{i}}{\partial x^{i}}\left(f_{i}(x, t), t\right) \\
& +\frac{1}{s(x, t)} \sum_{i} \frac{s_{i}\left(f_{i}(x, t)\right)}{s(x, t)}\left(\frac{\partial s_{i}}{\partial x^{i}}\left(f_{i}(x, t), t\right)-\frac{\partial s}{\partial x}(x, t)\right)\left(r_{i}\left(f_{i}(x, t)\right)-r(x, t)\right) .
\end{aligned}
$$

Just as Theorem 1, this theorem tells us that the change in the representative consumer's impatience can be decomposed into two terms. The first term on the right-hand side is easy to grasp. By (4), the first term can be rewritten as

$$
\sum_{i} \frac{s_{i}\left(f_{i}(x, t), t\right)}{s(x, t)}\left(\frac{\partial r_{i}}{\partial x^{i}}\left(f_{i}(x, t), t\right) \frac{\partial f_{i}}{\partial x}(x, t)\right)
$$

Here, the term $\left(\partial r_{i}\left(f_{i}(x, t), t\right) / \partial x^{i}\right)\left(\partial f_{i}(x, t) / \partial x\right)$ is the change in the impatience of consumer $i$ arising from the increase in his consumption level, which, in turn, caused by an increase in the aggregate consumption level. Thus the first term represents the direct effect on the representative consumer's impatience by the change in aggregate consumption. It is equal to zero when all individual consumers' felicity functions $u_{i}$ are multiplicatively separable.

The second term is equal to the weighted covariance, divided by the representative consumer's risk tolerance, between the individual consumers' cautiousness and impatience, where the weights are proportional to the individual consumers' risk tolerance. Since the second term would be zero if all consumers' cautiousness or impatience are equal to one another, it captures the tendency of changes in the representative consumer's impatience that arise from the heterogeneity in the individual consumers' cautiousness and impatience. Theorem 2 states that the change in the representative consumer's impatience is the sum of the individual counterparts, added by the weighted covariance, divided by his own risk tolerance, between the individual consumers' cautiousness and impatience.

Proof of Theorem 2 By (6),

$$
s(x, t) r(x, t)=\sum_{i} s_{i}\left(f_{i}(x, t), t\right) r_{i}\left(f_{i}(x, t), t\right)
$$


Differentiate both sides of (11) with respect to $x$, then we obtain

$$
\begin{aligned}
& \frac{\partial s}{\partial x}(x, t) r(x, t)+s(x, t) \frac{\partial r}{\partial x}(x, t) \\
= & \sum_{i}\left(\frac{\partial s_{i}}{\partial x^{i}}\left(f_{i}(x, t), t\right) \frac{\partial f_{i}}{\partial x}(x, t) r_{i}\left(f_{i}(x, t), t\right)+s_{i}\left(f_{i}(x, t), t\right) \frac{\partial r_{i}}{\partial x^{i}}\left(f_{i}(x, t), t\right) \frac{\partial f_{i}}{\partial x}(x, t)\right) .
\end{aligned}
$$

Thus

$$
\begin{aligned}
r(x, t)= & \frac{1}{s(x, t)} \sum_{i} s_{i}\left(f_{i}(x, t), t\right) \frac{\partial r_{i}}{\partial x^{i}}\left(f_{i}(x, t), t\right) \frac{\partial f_{i}}{\partial x}(x, t) \\
& +\frac{1}{s(x, t)}\left(\sum_{i} \frac{\partial s_{i}}{\partial x^{i}}\left(f_{i}(x, t), t\right) \frac{\partial f_{i}}{\partial x}(x, t) r_{i}\left(f_{i}(x, t), t\right)-\frac{\partial s}{\partial x}(x, t) r(x, t)\right) \\
= & \sum_{i}\left(\frac{s_{i}\left(f_{i}(x, t), t\right)}{s(x, t)}\right)^{2} \frac{\partial r_{i}}{\partial x^{i}}\left(f_{i}(x, t), t\right) \\
& +\frac{1}{s(x, t)}\left(\sum_{i} \frac{s_{i}\left(f_{i}(x, t), t\right)}{s(x, t)} \frac{\partial s_{i}}{\partial x^{i}}\left(f_{i}(x, t), t\right) r_{i}\left(f_{i}(x, t), t\right)-\frac{\partial s}{\partial x}(x, t) r(x, t)\right),
\end{aligned}
$$

where the last equality follows from (4). By (5) and (6),

$$
\begin{aligned}
& \sum_{i} \frac{s_{i}\left(f_{i}(x, t), t\right)}{s(x, t)} \frac{\partial s_{i}}{\partial x^{i}}\left(f_{i}(x, t), t\right) r_{i}\left(f_{i}(x, t), t\right)-\frac{\partial s}{\partial x}(x, t) r(x, t) \\
= & \sum_{i} \frac{s_{i}\left(f_{i}(x, t)\right)}{s(x, t)}\left(\frac{\partial s_{i}}{\partial x^{i}}\left(f_{i}(x, t), t\right)-\frac{\partial s}{\partial x}(x, t)\right)\left(r_{i}\left(f_{i}(x, t)\right)-r(x, t)\right) .
\end{aligned}
$$

The proof is thus completed.

In the case of multiplicatively separable utility functions, we have the following corollary.

Corollary 2 Suppose that $u_{i}$ is multiplicatively separable for every $i$. Then

$$
\frac{\partial r}{\partial x}(x, t)=\frac{1}{s(x, t)} \sum_{i} \frac{s_{i}\left(f_{i}(x)\right)}{s(x, t)}\left(s_{i}^{\prime}\left(f_{i}(x, t)\right)-\frac{\partial s}{\partial x}(x, t)\right)\left(r_{i}(t)-r(x, t)\right)
$$

for every $(x, t) \in \boldsymbol{R}_{++} \times \boldsymbol{R}_{+}$. Moreover,

1. If $\left(s_{1}^{\prime}\left(f_{1}(x, t)\right), \ldots, s_{I}^{\prime}\left(f_{I}(x, t)\right)\right)$ and $\left(r_{1}(t), \ldots, r_{I}(t)\right)$ are comonotone (that is, $\left(s_{i}^{\prime}\left(f_{i}(x, t)\right)-s_{j}^{\prime}\left(f_{j}(x, t)\right)\right)\left(r_{i}(t)-r_{j}(t)\right) \geq 0$ for every pair of two consumers $i$ and $\left.j\right)$, then $\partial r(x, t) / \partial x \geq 0$. This weak inequality holds as an equality if and only if $s_{1}^{\prime}\left(f_{1}(x, t)\right)=$ $\cdots=s_{I}^{\prime}\left(f_{I}(x, t)\right)$ or $r_{1}(t)=\cdots=r_{I}(t)$.

2. If $\left(s_{1}^{\prime}\left(f_{1}(x, t)\right), \ldots, s_{I}^{\prime}\left(f_{I}(x, t)\right)\right)$ and $\left(r_{1}(t), \ldots, r_{I}(t)\right)$ are anti-comonotone (that is, $\left(s_{i}^{\prime}\left(f_{i}(x, t)\right)-s_{j}^{\prime}\left(f_{j}(x, t)\right)\right)\left(r_{i}(t)-r_{j}(t)\right) \leq 0$ for every pair of two consumers $i$ and $\left.j\right)$, then $\partial r(x, t) / \partial x \leq 0$. This weak inequality holds as an equality if and only if $s_{1}^{\prime}\left(f_{1}(x, t)\right)=$ $\cdots=s_{I}^{\prime}\left(f_{I}(x, t)\right)$ or $r_{1}(t)=\cdots=r_{I}(t)$. 
We next give a formula for $\partial r(x, t) / \partial t$, which shows how the representative consumer's impatience varies over time.

Theorem 3 For every $(x, t) \in \boldsymbol{R}_{++} \times \boldsymbol{R}_{+}$,

$$
\begin{aligned}
\frac{\partial r}{\partial t}(x, t)= & \sum_{i} \frac{s_{i}\left(f_{i}(x, t), t\right)}{s(x, t)} \frac{\partial r_{i}}{\partial t}\left(f_{i}(x, t), t\right) \\
& +\sum_{i} \frac{s_{i}\left(f_{i}(x, t), t\right)}{s(x, t)}\left(r_{i}\left(f_{i}(x, t), t\right)-r(x, t)\right)^{2} \frac{\partial s_{i}}{\partial x^{i}}\left(f_{i}(x, t), t\right) \\
& +\sum_{i} \frac{s_{i}\left(f_{i}(x, t), t\right)}{s(x, t)}\left(r_{i}\left(f_{i}(x, t), t\right)-r(x, t)\right) \\
& \times\left(\frac{\frac{\partial s_{i}}{\partial t}\left(f_{i}(x, t), t\right)}{s_{i}\left(f_{i}(x, t), t\right)}-\frac{\frac{\partial s}{\partial t}(x, t)}{s(x, t)}+\frac{\partial r_{i}}{\partial x^{i}}\left(f_{i}(x, t), t\right) s_{i}\left(f_{i}(x, t), t\right)\right) .
\end{aligned}
$$

This theorem tells us that the rate, per unit of time, of changes in the representative consumer's impatience can be decomposed into three terms. The first term is easy to grasp. As shown by (6), the representative consumer's impatience is equal to the weighted average of the individual consumers' counterparts, where the weights are proportional to their risk tolerance. Thus the first term represents the direct effect, by time, on the representative consumer's impatience, while the weights are hypothetically fixed. It is equal to zero if all the consumers' felicity functions $u_{i}$ are of exponential discounting.

The third term represents the change in the representative consumer's impatience caused by the impact on the individual consumers' risk tolerance by time, and also by the impact on their impatience by consumption levels. It is equal to zero if all consumers' felicity functions are multiplicatively separable.

The second term is most interesting. It represents the impact on the representative consumer's impatience when the individual consumers have differing impatience. As mentioned above, the representative consumer's impatience is equal to the weighted average of the individual consumers' counterparts, and the weights are proportional to their risk tolerance. If their impatience are different, then the risk-sharing rules $f_{i}$ would depend on time $t$; that is, the partial derivative $\partial f_{i}(x, t) / \partial t$ would be different from zero. Unless the cautiousness, $\partial s_{i}\left(f_{i}(x, t), t\right) / \partial x_{i}$, is zero (which would be the case if $u_{i}$ exhibited constant absolute, rather than relative, risk aversion), the change in consumption levels has an impact on the individual consumers' risk tolerance, and thus on the representative consumer's impatience, which is the weighted average of the individual consumers' impatience, with the weights given by their risk tolerance. The second term, therefore, captures the change in the representative consumer's impatience arising from the heterogeneity in the individual consumers' impatience.

Proof of Theorem 3 By (6) and differentiation for a product,

$$
\frac{\partial r}{\partial t}(x, t)=\sum_{i} \frac{d}{d t}\left(\frac{s_{i}\left(f_{i}(x, t), t\right)}{s(x, t)}\right) r_{i}\left(f_{i}(x, t), t\right)+\sum_{i} \frac{s_{i}\left(f_{i}(x, t), t\right)}{s(x, t)} \frac{d}{d t}\left(r_{i}\left(f_{i}(x, t), t\right)\right) .
$$


By (7),

$$
\begin{aligned}
& \frac{d}{d t}\left(\frac{s_{i}\left(f_{i}(x, t), t\right)}{s(x, t)}\right) \\
= & \frac{\frac{\partial s_{i}}{\partial x^{i}}\left(f_{i}(x, t), t\right)}{s(x, t)} \frac{\partial f_{i}}{\partial t}(x, t)+\frac{\frac{\partial s_{i}}{\partial t}\left(f_{i}(x, t), t\right)}{s(x, t)}-\frac{s_{i}\left(f_{i}(x, t), t\right)}{(s(x, t))^{2}} \frac{\partial s}{\partial t}(x, t) \\
= & \frac{s_{i}\left(f_{i}(x, t), t\right)}{s(x, t)}\left(\frac{\frac{\partial s_{i}}{\partial t}\left(f_{i}(x, t), t\right)}{s_{i}\left(f_{i}(x, t), t\right)}-\frac{\frac{\partial s}{\partial t}(x, t)}{s(x, t)}+\frac{\partial s_{i}}{\partial x^{i}}\left(f_{i}(x, t), t\right)\left(r_{i}\left(f_{i}(x, t), t\right)-r(x, t)\right)\right) .
\end{aligned}
$$

By (3),

$$
\sum_{i} \frac{d}{d t}\left(\frac{s_{i}\left(f_{i}(x, t), t\right)}{s(x, t)}\right)=0 .
$$

Thus,

$$
\begin{aligned}
& \sum_{i} \frac{d}{d t}\left(\frac{s_{i}\left(f_{i}(x, t), t\right)}{s(x, t)}\right) r_{i}\left(f_{i}(x, t), t\right) \\
= & \sum_{i} \frac{d}{d t}\left(\frac{s_{i}\left(f_{i}(x, t), t\right)}{s(x, t)}\right)\left(r_{i}\left(f_{i}(x, t), t\right)-r(x, t)\right) \\
= & \sum_{i} \frac{s_{i}\left(f_{i}(x, t), t\right)}{s(x, t)} \frac{\partial s_{i}}{\partial x^{i}}\left(f_{i}(x, t), t\right)\left(r_{i}\left(f_{i}(x, t), t\right)-r(x, t)\right)^{2} \\
& +\sum_{i} \frac{s_{i}\left(f_{i}(x, t), t\right)}{s(x, t)}\left(\frac{\frac{\partial s_{i}}{\partial t}\left(f_{i}(x, t), t\right)}{s_{i}\left(f_{i}(x, t), t\right)}-\frac{\frac{\partial s}{\partial t}(x, t)}{s(x, t)}\right)\left(r_{i}\left(f_{i}(x, t), t\right)-r(x, t)\right)
\end{aligned}
$$

Again by (7),

$$
\begin{aligned}
& \frac{d}{d t}\left(r_{i}\left(f_{i}(x, t), t\right)\right) \\
= & \frac{\partial r_{i}}{\partial x^{i}}\left(f_{i}(x, t), t\right) \frac{\partial f_{i}}{\partial t}(x, t)+\frac{\partial r_{i}}{\partial t}\left(f_{i}(x, t), t\right) \\
= & \frac{\partial r_{i}}{\partial x^{i}}\left(f_{i}(x, t), t\right) s_{i}\left(f_{i}(x, t), t\right)\left(r_{i}\left(f_{i}(x, t), t\right)-r(x, t)\right)+\frac{\partial r_{i}}{\partial t}\left(f_{i}(x, t), t\right) .
\end{aligned}
$$

Hence,

$$
\begin{aligned}
& \sum_{i} \frac{s_{i}\left(f_{i}(x, t), t\right)}{s(x, t)} \frac{d}{d t}\left(r_{i}\left(f_{i}(x, t), t\right)\right) \\
= & \sum_{i} \frac{s_{i}\left(f_{i}(x, t), t\right)}{s(x, t)} \frac{\partial r_{i}}{\partial t}\left(f_{i}(x, t), t\right) \\
& +\sum_{i} \frac{s_{i}\left(f_{i}(x, t), t\right)}{s(x, t)} \frac{\partial r_{i}}{\partial x^{i}}\left(f_{i}(x, t), t\right) s_{i}\left(f_{i}(x, t), t\right)\left(r_{i}\left(f_{i}(x, t), t\right)-r(x, t)\right) .
\end{aligned}
$$

Thus, by (13), (14), and (15), we obtain (12). 
The right-hand side of (12) in Theorem 3 can be much simplified if we concentrate on the case of multiplicatively separable felicity functions. The following corollary follows from Theorem 3 and $(6)$

Corollary 3 Suppose that $u_{i}$ is multiplicatively separable for every $i$. Then

$$
\frac{\partial r}{\partial t}(x, t)=\sum_{i} \frac{s_{i}\left(f_{i}(x, t)\right)}{s(x, t)} r_{i}^{\prime}(t)-\sum_{i} \frac{s_{i}\left(f_{i}(x, t)\right)}{s(x, t)}\left(r_{i}(t)-r(x, t)\right)^{2} s_{i}^{\prime}\left(f_{i}(x, t)\right)
$$

for every $(x, t) \in \boldsymbol{R}_{++} \times \boldsymbol{R}_{+}$. Moreover,

1. If $r^{\prime}(t) \leq 0$ and $s_{i}^{\prime}\left(f_{i}(x, t)\right) \geq 0$ for every $i$, then $\partial r(x, t) / \partial t \leq 0$. This weak inequality holds as an equality if and only if $r_{1}^{\prime}(t)=\cdots=r_{I}^{\prime}(t)=0$ and, in addition, either $r_{1}(t)=$ $\cdots=r_{I}(t)$ or $s_{1}^{\prime}\left(f_{1}(x, t)\right)=\cdots=s_{I}^{\prime}\left(f_{I}(x, t)\right)=0$.

2. If $r^{\prime}(t) \geq 0$ and $s_{i}^{\prime}\left(f_{i}(x, t)\right) \leq 0$ for every $i$, then $\partial r(x, t) / \partial t \geq 0$. This weak inequality holds as an equality if and only if $r_{1}^{\prime}(t)=\cdots=r_{I}^{\prime}(t)=0$ and, in addition, either $r_{1}(t)=$ $\cdots=r_{I}(t)$ or $s_{1}^{\prime}\left(f_{1}(x, t)\right)=\cdots=s_{I}^{\prime}\left(f_{I}(x, t)\right)=0$.

\section{Conclusion}

We have investigated implications of heterogeneous impatience in an economy populated by multiple consumers who have time-separable utility functions. We have found some formulas showing how the representative consumer's risk attitudes will change over time and how the change in aggregate consumption levels affect his impatience. These formulas clarify when and, if so, how, his felicity function fails to be multiplicatively separable. We have also found a formula showing how his impatience will change over time. This formula clarifies how the change in his impatience is determined by the heterogeneity of individual consumers' impatience and cautiousness.

Based on these results, we have characterized the representative consumer's discount factor, using a property called complete monotonicity. We have also applied these results to derive some properties of the short-rate process in an economy of heterogeneous consumers.

There are some issued yet to be explored in this setting. First, while we concentrated on the case of time-additive utility functions, we should look into whether there is any coherence of our analysis with recursive or stochastic differential utility functions. ${ }^{3}$ These utility functions are in general not time-additive, but they are still tractable and useful for many applications. It would therefore be reasonable to try to extend our analysis to these utility functions. Second, we have not investigated whether the heterogeneity of impatience would give rise to most commonly used properties in the literature on the term structure of interest rates. One of such properties is mean reversion, so that the drift term of the short-rate process will become negative if the short rate exceeds some threshold, while it will become positive if the short rate goes below

\footnotetext{
${ }^{3}$ I am grateful to Tomoyuki Nakajima for suggesting this line of research.
} 
the threshold. It will quite important to identify under what conditions of heterogeneity of impatience the short-rate process would be mean-reverting. ${ }^{4}$

\section{References}

[1] Patrick Billingsley, 1995, Probability and Measure, 3rd ed, John Wiley and Sons, New York.

[2] Christian Gollier and Richard Zeckhauser, Aggregation of heterogeneous time preferences, Journal of Political Economy 113 (2005), 878-896.

[3] Chiaki Hara, 2006, Heterogeneous risk attitudes in a continuous-time model, Japanese Economic Review 57 (2006), 377-405.

[4] Chiaki Hara, James Huang, and Christoph Kuzmics, 2007, Efficient risk-sharing rules and the representative consumer's risk aversion, forthcoming in Journal of Economic Theory.

[5] Semyon Malamud and Eugene Trubowitz, Aggregate risk aversion for jointly heterogeneous discount factors and risk tolerances, working paper.

\footnotetext{
${ }^{4}$ I am grateful to Masaaki Kijima for suggesting this line of research.
} 